\title{
Neuroprotective effects of mGluR5 activation through the PI3K/Akt pathway and the molecular switch of AMPA receptors
}

\author{
Damiana Cavallo $^{\mathrm{a}, \mathrm{b}, *, 1}$, Elisa Landucci ${ }^{\mathrm{a}}$, Elisabetta Gerace ${ }^{\mathrm{a}}$, Daniele Lana ${ }^{\mathrm{a}}$, Filippo Ugolini ${ }^{\mathrm{a}}$, \\ Jeremy M. Henley ${ }^{\mathrm{b}}$, Maria Grazia Giovannini ${ }^{\mathrm{a}}$, Domenico E. Pellegrini-Giampietro ${ }^{\mathrm{a}}$ \\ a Department of Health Sciences, Unit of Clinical Pharmacology and Oncology, University of Florence, Viale G. Pieraccini 6, 50139, Florence, Italy \\ ${ }^{\mathrm{b}}$ School of Biochemistry, Centre for Synaptic Plasticity, Biomedical Sciences Building, University of Bristol, University Walk, Bristol, BS8 1TD, UK
}

H I G H L I G H T S

- mGluR5 activation with DHPG and VU0092273 showed neuroprotective effects in OGD.

- mGluR5 exerted its neuroprotective effects via PI3K/Akt pathway.

- mGluR5 also prevented the GluA2 down-regulation following an ischemic injury.

- mGluR5 appears to stimulate neuroprotective responses in astrocytes and microglia.

\section{A R T I C L E I N F O}

\section{Keywords:}

Metabotropic glutamate receptors

Positive allosteric modulators (PAMs)

PI3K/Akt

GluA2 subunit

Astrocytes

Microglia

Oxygen-glucose deprivation (OGD)

\begin{abstract}
A B S T R A C T
Previous studies have demonstrated that antagonists of mGluR1, but not mGluR5, are neuroprotective in models of cerebral ischemia. To investigate the individual roles of mGlu1 and mGlu5 receptors in in vitro model of cerebral ischemia we used low doses of the non-selective group I agonist DHPG and mGlu1 and mGlu5 selective positive allosteric modulators (PAMs). In hippocampal slices subjected to $30 \mathrm{~min}$ oxygen-glucose deprivation (OGD), DHPG $(1 \mu \mathrm{M})$ and the mGluR5 PAM (VU0092273) significantly reduced OGD-induced CA1 injury monitored by propidium iodide staining of the slices and quantitative analysis of CA1 neurons. In contrast, the mGluR1 PAM (VU0483605) showed no neuroprotection. These protective effects of DHPG and VU0092273 were prevented by inhibition of PI3K/Akt pathway by LY294002. The mGluR5 PAM (VU0092273) also prevented GluA2 down-regulation triggered by ischemic injury, via PI3K/Akt pathway, revealing a further contribution to its neuroprotective effects by reducing the excitotoxic effects of increased $\mathrm{Ca}^{2+}$ influx through GluA2-lacking AMPA receptors. Furthermore, immunohistochemical assays confirmed the neuroprotective effect of VU0092273 and revealed activation of glia, indicating the involvement reactive astrogliosis in the mechanisms of neuroprotection. Our data suggest that selective activation/potentiation of mGluR5 signalling represents a promising strategy for the development of new interventions to reduce or prevent ischemia-induced neuronal death.
\end{abstract}

\section{Introduction}

Ischemia caused by stroke is one of the main causes of mortality and long-term disability worldwide (Law et al., 2017). Massive glutamate release following an ischemic injury triggers an excitotoxic cascade, which leads to glutamate receptor-mediated delayed cell death of vulnerable neuronal populations (Buchan and Pulsinelli, 1990).

Metabotropic glutamate (mGlu) receptors are classified into three subgroups (Groups I, II and III) based on sequence homology, signalling transduction pathways and relative pharmacology (Conn and Pin, 1997). Of these, group I comprises mGlu1 and mGlu5, which coupled to $\mathrm{G}_{\mathrm{q} / 11}$ and mediate IP3/ $/ \mathrm{Ca}^{2+}$ signal transduction via phospholipase $\mathrm{C}$ (PLC) activation (Abe et al., 1992). Although the two subtypes of group I metabotropic glutamate receptors are highly homologous, they play different roles in the cerebral ischemia. mGlu1, but not mGlu5, antagonists are neuroprotective in models of cerebral ischemia (Pellegrini-

\footnotetext{
* Corresponding author. School of Physiology, Pharmacology and Neuroscience, Faculty of Life Sciences, Biomedical Sciences Building, University of Bristol, University Walk, Bristol, BS8 1TD, UK.

E-mail address: damiana.cavallo@bristol.ac.uk (D. Cavallo).

${ }^{1}$ Present address: School of Physiology, Pharmacology and Neuroscience, Faculty of Life Sciences, Biomedical Sciences Building, University Walk, Bristol, BS8 1TD (UK).
} 


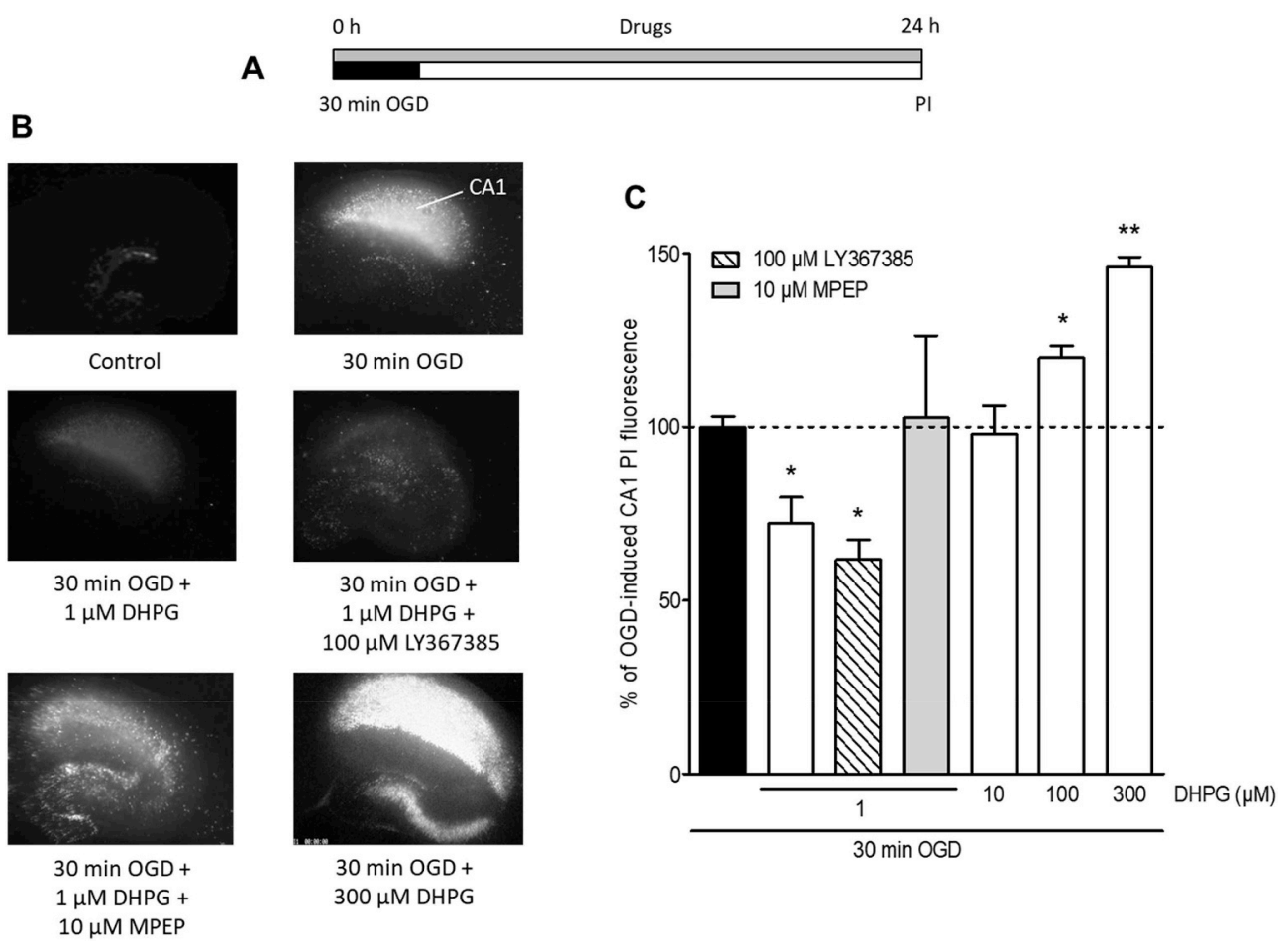

Fig. 1. Dose-dependent effects of DHPG against OGD injury in rat organotypic hippocampal slices. (A) Schematic diagram showing that slices were incubated with mGlu receptor agonists and antagonists during OGD and the subsequent recovery period. Twenty-four $h$ later slices were incubated with PI for fluorescence detection of their optical density. (B) Hippocampal slices, photographed under fluorescence optics, displaying background levels of fluorescence under control conditions, an intense PI labeling in the CA1 sub-region $24 \mathrm{~h}$ after exposure to $30 \mathrm{~min}$ OGD, a reduction in PI fluorescence when incubated with $1 \mu \mathrm{M}$ DHPG, that was prevented by the mGlu5 antagonist MPEP but not by the mGlu1 antagonist LY367385, and a marked increase in OGD-induced PI fluorescence when incubated with $300 \mu \mathrm{M}$ DHPG. (C) Quantitative analysis of OGD-induced CA1 PI fluorescence expressed as percentage of OGD toxicity. Incubation with $1 \mu \mathrm{M}$ DHPG significantly attenuated OGD injury; the effect was completely reverted by the coincubation with the mGlu5 antagonist MPEP $(10 \mu \mathrm{M})$ but not with the mGlu1 antagonist LY367385 $(100 \mu \mathrm{M})$. Incubation with $10 \mu \mathrm{M}$ DHPG had no effect whereas

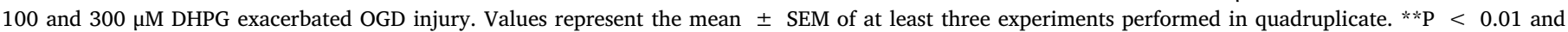
${ }^{*} \mathrm{P}<0.05$ vs. $30 \mathrm{~min}$ OGD alone (ANOVA + Tukey's w-test).

Giampietro, 2003). In addition, mGlu5 is expressed on astrocytes where it contributes to the modulation of synaptic transmission efficacy and local blood flow (Arizono et al., 2014) and on microglia where it mediates numerous actions essential for intrinsic glial cell function, as well as for glial-neuronal interactions (Loane et al., 2012).

Positive allosteric modulators, PAMs, are compounds that interact at "allosteric" sites, topographically distinct from the orthosteric site, where they can affect the affinity and/or efficacy of an orthosteric ligand, a property referred to as cooperativity. This is useful because the allosteric site contains a higher level of sequence diversity between receptor subtypes than the orthosteric site, allowing for greater subtype selectivity of allosteric ligands (Christopoulos and Kenakin, 2002; Conn et al., 2009). Moreover, allosteric agents have the potential to show differential effects on downstream signalling pathways, termed functional selectivity. Thus, PAMs provide a potential opportunity to develop agents that selectively target GPCR signalling pathways critical for therapeutic efficacy without modulating signalling pathways that lead to adverse effects (Nickols and Conn, 2014).

To better discriminate the role of group I metabotropic glutamate receptors we used the non-selective agonist DHPG and two selective positive allosteric modulators (PAMs), mGluR1 PAM 3-chloro- $N$-(3chloro-4-(4-chloro-1,3-dioxoioindolin-2-yl)picolinamide, VU0483605, and the mGluR5 PAM (4-hidroxypiperidin-1-yl)(4-(phenylethynyl) phenyl)methanone, VU0092273, in the in vitro model of OGD. Our results show that selectively enhancing mGluR5 signalling can be neuroprotective against neuronal death in CA1 via a mechanism that involves both activation of the PI3K/Akt pathway and the prevention of the GluA2 AMPA subunit down-regulation induced by OGD.

\section{Materials and methods}

Experiments and animal use procedures were in accordance with the National Institutes of Health Guide for the Care and Use of Laboratory Animals (NIH Publications No. 80-23, revised 1996). The experimental protocols were approved by the Animal Committee of the Department of Pharmacology, University of Florence, in compliance with the European Convention for the Protection of Vertebrate Animals used for Experimental and Other Scientific Purposes (ETS No. 123) and the European Communities Council Directive of 24 November 1986 (86/609/EEC). The authors further attest that all efforts were made to minimize the number of animals used and their suffering.

\subsection{Materials}

(S)-3,5-Dihydroxyphenylglycine (DHPG), (S)-(+)- $\alpha$-amino-4-carboxy-2-methylbenzeneacetic acid (LY367385) and 2-methyl-6-(phenylethynyl)pyridine hydrochloride (MPEP) were purchased from Tocris Cookson (Bristol, UK). 1-Naphthylacetyl spermine trihydrochloride (NASPM) was purchased from Sigma-Aldrich, St. Louis, MO, USA). 3Chloro- $N$-[3-chloro-4-(4-chloro-1,3-dihydro-1,3-dioxo-2H-isoindol-2yl)phenyl]-2-pyridinecarboxamide (VU0483605) and (4-hydroxypiperidin-1-yl)-[4-(2-phenylethynyl)phenyl]methanone

(VU0092273) were generously provided by Dr. Jeffrey P. Conn (Vanderbilt University Medical Center, Nashville, TN, USA). 2-(4-Morpholinyl)-8-(4-aminophenyl)-4H-1-benzopyrone-4-one (LY 294002) was purchased from Calbiochem (Merck Biosciences Ltd, Nottingham, UK). Propidium iodide (PI) was purchased from Molecular Probes (Leiden, the Netherlands) and tissue culture reagents were obtained from Gibco-BRL (San Giuliano Milanese, MI, Italy) and Sigma (St Louis, MO, USA).

\subsection{Oxygen-glucose deprivation in organotypic hippocampal slice cultures}

Organotypic hippocampal slice cultures were prepared as previously reported in detail (Gerace et al., 2012). Briefly, hippocampi were removed from the brains of 7-9 days old Wistar rats (Harlan, MI, Italy), and transverse slices $(420 \mu \mathrm{m})$ were prepared using a McIlwain tissue chopper. Slices were then transferred onto $30 \mathrm{~mm}$ diameter semiporous membranes inserts (Millicell-CM PIC M 03050; Millipore, Italy), which were placed in six-well tissue culture plates containing $1.2 \mathrm{ml}$ culture medium per well. Slices were maintained at $37^{\circ} \mathrm{C}$ in an incubator in atmosphere of humidified air and 5\% $\mathrm{CO} 2$ and the culture medium was 
changed thrice a week. Experiments were carried out after 14 days in vitro. The slices were subjected to OGD by exposing them to a serumand glucose-free medium saturated with $95 \% \mathrm{~N} 2$ and $5 \% \mathrm{CO} 2$ at $37{ }^{\circ} \mathrm{C}$ in an airtight anoxic chamber equipped with an oxygen gas controller (BioSpherix, New York, USA). After $30 \mathrm{~min}$, the cultures were transferred to oxygenated serum-free medium containing $5 \mathrm{mg} / \mathrm{ml}$ glucose and maintained at $37^{\circ} \mathrm{C}, 35^{\circ} \mathrm{C}$ or $32^{\circ} \mathrm{C}$ in different incubators under normoxic conditions until neuronal injury was evaluated $24 \mathrm{~h}$ later. Drugs were present in the incubation medium during OGD and the subsequent $24 \mathrm{~h}$ recovery period (Fig. 1A). Cell injury was assessed by using the fluorescent dye PI $(5 \mu \mathrm{g} / \mathrm{ml})$; fluorescence was viewed using an inverted fluorescence microscope. Images were digitized using a video image controlled by software and subsequently analysed using a morphometric analysis software. In order to quantify cell death, the CA1 hippocampal subfield was identified and encompassed in a frame using the drawing function in the image software (ImageJ; NIH, Bethesda, USA) and the optical density of PI fluorescence was detected. Control experiments performed in our laboratory, as well as a number of previous studies using organotypic hippocampal slices exposed to ischemia-related insults, have shown that there is a linear correlation between relative PI fluorescence and the number of injured cells as detected by morphological criteria (Gerace et al., 2012).

\subsection{Western blot analysis}

Hippocampal slices were washed with cold $0.01 \mathrm{M}$ phosphate-buffered saline, $\mathrm{pH} 7.4$ and 4 slices/sample were gently transferred and dissolved in a tube containing $1 \%$ sodium dodecyl sulfate (SDS) as previously reported in detail (Gerace et al., 2016; Landucci et al., 2016). Total protein levels were quantified using the Pierce (Rockford, IL, USA) BCA (bicinchoninic acid) Protein Assay. Homogenates (40 $\mu \mathrm{g}$ of protein) were resolved by electrophoresis on 4-20\% (wt/vol) SDSpolyacrylamide gel (Bio-Rad Laboratories, Hercules, CA, USA) and transferred onto nitrocellulose membranes using the transblot TURBO (Bio-Rad, Hercules, CA, USA). Blots were blocked for $1 \mathrm{~h}$ at room temperature in Tris-buffered saline, $\mathrm{pH} 7.6,0.1 \%$ Tween 20 (TBS-T) containing 5\% non-fat dry milk, and then incubated overnight at $4{ }^{\circ} \mathrm{C}$ with a polyclonal rabbit antibody directed against phospho-Akt (Ser473) or phospho-GSK3- $\beta$ (Ser9) (all from Cell Signalling Technology, Beverly, MA, USA) diluted 1:1000 in TBS-T containing 5\% bovine serum albumin and polyclonal goat anti-GluA2 (Santa Cruz Biotechnology, Paso Robles, CA, USA) diluted 1:1000 in TBS-T containing non-fat dry milk. Monoclonal $\alpha$-tubulin antibody (Sigma, 1:5000) was used as loading control. Immunodetection was performed with secondary antibodies (1:2000 anti-rabbit, anti-goat and antimouse IgG from donkey; Amersham Biosciences, Buckinghamshire, UK)) conjugated to horseradish peroxidase. The reactive bands were detected using chemiluminescence (ECLplus; Euroclone, Padova, Italy). Quantitative analysis was performed using the QuantityOne analysis software (Bio-Rad, Hercules, CA, USA).

\subsection{Triple labelling immunohistochemistry}

Organotypic hippocampal slices were triple immunostained with the free floating method as described (Lana et al., 2014). Briefly, after blocking with Blocking Buffer (BB), slices were incubated overnight under agitation with the following primary antibodies: a mouse monoclonal anti-neuronal nuclei (NeuN, 1:200, Millipore, Billerica, MA, USA) for neurons, and a rabbit polyclonal anti IBA1, (1:300, WAKO, Osaka, Japan) for microglia, both diluted in BB solution. IBA1 (Ionized calcium binding adaptor molecule 1 ) is an intracellular $17-\mathrm{kDa}$ protein whose expression is restricted to microglia/macrophages (Imai et al., 1996) Iba1 is a protein for cytoskeletal rearrangement in microglia, widely used as a specific marker for total microglia. All the following steps were done at room temperature in the dark for $2 \mathrm{~h}$. After washings, slices were incubated with an anti-rabbit secondary antibody conjugated with AlexaFluor 635 (for microglia, 1:400, Thermo Fisher Sci., Waltham, MA, USA). After incubation, a secondary antibody conjugated with AlexaFluor 555 (for neurons, 1:400, Thermo Fisher Sci.) was added, and slices were incubated as above. Finally, astrocytes were immunostained with a primary monoclonal anti GFAP antibody conjugated with AlexaFluor 488 (1:500, Millipore). Glial Fibrillary Acidic Protein (GFAP), is a structural protein (Bignami et al., 1972) a key component of the astrocyte's cytoskeleton, and classically used as a marker for astrocytes (Middeldorp and Hol, 2011). After washings, the slices were mounted with Vectashield mounting medium with DAPI (Vector Laboratories, Burlingame, CA, USA).

\subsection{Confocal laser scanning microscopy and quantitative analysis}

Images were acquired under a LEICA TCS SP5 confocal laser scanning microscope (Leica Microsystems CMS GmbH, Mannheim, Germany) with a 20X objective. Confocal scans were taken at $1 \mu \mathrm{m} z$ steps keeping all parameters (pinhole, contrast and brightness) constant. Image analyses were conducted on z-stacks which contained the region of interest, corresponding to the CA1 area. Images were digitally converted to green, red or blue using Image $J$ (National Institute of Health, http://rsb.info.nih.gov/ij).

Quantification of astrocytes, neurons and microglia immunofluorescence was performed separately using Image J. Three consecutive z-scans ( $1 \mu \mathrm{m}$ each, total $3 \mu \mathrm{m}$ ) were stacked, starting at $10 \mu \mathrm{m}$ inside the slice and an appropriate and constant threshold level was selected. Quantitation of immunofluorescence of NeuN, GFAP or IBA1 was then obtained from the ratio between positive pixels above threshold and total pixels in each region of interest.

\subsection{Statistical analysis}

Data are presented as means \pm SEM of $n$ experiments. Statistical significance of differences were analysed using one-way ANOVA followed by the post hoc Dunnet and Tukey's w-test for multiple comparisons. All statistical calculations were performed using GRAPH-PAD PRISM v. 5 for Windows (GraphPad Software, San Diego, CA, USA). A probability value $(\mathrm{P})$ of $<0.05$ was considered significant.

\section{Results}

\subsection{Activation of mGlu5 receptors is neuroprotective in rat organotypic hippocampal slices exposed to OGD}

We have reported previously that the nonselective group I mGlu receptor agonist DHPG at 100 and $300 \mu \mathrm{M}$ exacerbates OGD injury in organotypic hippocampal slices (Pellegrini-Giampietro et al., 1999) and that this toxic effect is most likely mediated by mGlu1 rather than mGlu5 receptors (Pellegrini-Giampietro, 2003). On the other hand, we observed that DHPG induces ischemic tolerance at subtoxic $(<10 \mu \mathrm{M})$ concentrations in the same model via the contribution of both mGlu1 and mGlu5 receptors (Werner et al., 2007). Therefore, in this study examined whether low, subtoxic concentrations of DHPG could also protect organotypic hippocampal slices against OGD injury.

Consistent with our previous observations (Pellegrini-Giampietro et al., 1999; Meli et al., 2002), when relatively high concentrations of DHPG (100-300 $\mu \mathrm{M})$ were added to the incubation medium of hippocampal slices during OGD and the $24 \mathrm{~h}$ recovery period the damage in the CA1 region induced by simulated ischemia was significantly enhanced (Fig. 1). Conversely, a low concentration of DHPG (1 $\mu \mathrm{M})$ produced a $30 \%$ reduction in OGD injury that was completely prevented by the mGlu5 antagonist MPEP $(10 \mu \mathrm{M})$ but not by the mGlu1 antagonist LY367385 $(100 \mu \mathrm{M})$. An intermediate concentration of DHPG $(10 \mu \mathrm{M})$ was neither toxic nor neuroprotective under our experimental conditions.

To corroborate the contribution of mGlu5 receptors in the 
A

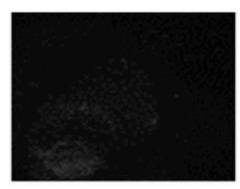

Control

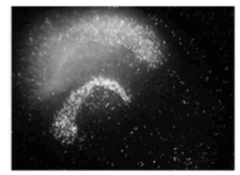

$30 \mathrm{~min} O G D+$ $1 \mu \mathrm{M}$ VU0483605

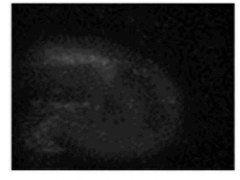

$30 \mathrm{~min} O G D+$ $10 \mathrm{nM}$ VU0092273
B

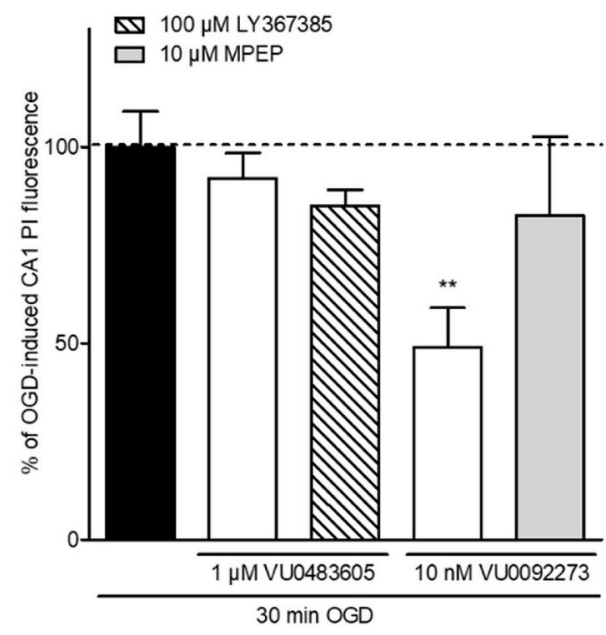

Fig. 2. The mGlu5 receptor PAM VU0092273 but not the mGlu1 receptor PAM VU0483605 attenuates OGD injury in hippocampal slices. The experiments were carried out and drugs were added as shown in Fig. 1A. (A) Hippocampal slices, photographed under fluorescence optics, displaying selective damage of the CA1 subregion $24 \mathrm{~h}$ following $30 \mathrm{~min}$ OGD and a reduction in PI fluorescence when incubated with the mGlu5 receptor PAM VU0092273, that was reverted by the mGlu5 antagonist MPEP, but not with the mGlu1 receptor PAM VU0483605. (B) Quantitative analysis of OGD-induced CA1 PI fluorescence expressed as percentage of OGD toxicity. Incubation with $10 \mathrm{nM}$ VU0092273 significantly attenuated OGD injury; the effect was reverted by co-incubation with the mGlu5 antagonist MPEP $(10 \mu \mathrm{M})$. The mGlu1 receptor PAM VU $0483605(1 \mu \mathrm{M})$ incubated alone or in combination with the mGlu1 antagonist LY367385 $(100 \mu \mathrm{M})$ had no effect on OGD injury. Values represent the mean \pm SEM of at least three experiments performed in quadruplicate. $* * \mathrm{P}<0.01$ vs. 30 min OGD alone (ANOVA $+\mathrm{Tu}-$ key's w-test).
$30 \mathrm{~min} O G D+$ $10 \mathrm{nM}$ VU0092273 + $10 \mu \mathrm{M}$ MPEP protective effects of DHPG, we compared the effects of VU0483605, a positive allosteric modulator (PAM) of mGlu1 receptors with an $\mathrm{EC}_{50}$ of $350 \mathrm{nM}$ (Cho et al., 2014), and VU0092273, a PAM of mGlu5 receptors with an $\mathrm{EC}_{50}$ of $10 \mathrm{nM}$ (Noetzel et al., 2012). In hippocampal slices exposed to $30 \mathrm{~min}$ OGD, the mGlu1 PAM VU0483605 $(1 \mu \mathrm{M})$ did not reduce OGD injury when present during the $30 \mathrm{~min}$ of OGD and the subsequent $24 \mathrm{~h}$ recovery period. Moreover, there was no effect of the mGlu1 antagonist LY367385 (100 $\mu \mathrm{M})$ (Fig. 2). On the other hand, the mGlu5 PAM VU0092273 (10 nM) reduced PI fluorescence in the CA1 area by approximately $50 \%$, and this protective effect was reversed by the mGlu5 antagonist MPEP (10 $\mu \mathrm{M})$ (Fig. 2).

\subsection{Effects of the activation of mGlu5 receptors on neurons, astrocytes and} microglia in the CA1 subregion of rat organotypic hippocampal slices exposed to $O G D$

We next assessed the effects of DHPG and VU0092273 on both CA1 pyramidal cell viability and astrocyte and microglia proliferation by using triple immunostaining for NeuN, GFAP and IBA1 on organotypic slices harvested $24 \mathrm{~h}$ after $30 \mathrm{~min}$ OGD, followed by confocal microscopy. Representative images are shown in Fig. 3 (red for neurons, green for astrocytes and blue for microglia) obtained from triple immunostaining experiments performed on the same slice exposed to control conditions (Fig. 3. A1-C1a), $30 \mathrm{~min}$ OGD (Fig. 3. A2-C2a), $30 \mathrm{~min}$ OGD in the presence of $1 \mu \mathrm{M}$ DHPG (Fig. 3. A3-C3a), and $30 \mathrm{~min}$ OGD in the presence of $10 \mathrm{nM}$ VU009227 (Fig. 3. A4-C4a).

NeuN immunostaining (Fig. 3. A1-A4 and 3D) confirmed that viability of CA1 pyramidal neurons exposed to $30 \mathrm{~min}$ OGD was significantly decreased (by $32 \%$ ) as compared to control slices. This loss of neurons was prevented by $1 \mu \mathrm{M}$ DHPG and $10 \mathrm{nM}$ VU0092273. Conversely, GFAP immunostaining (Fig. 3. B1-B4 and 3E) showed that astrocytes were not significantly affected $24 \mathrm{~h}$ after OGD injury. Quantitative analysis (Fig. 3E) demonstrated that both treatments with DHPG (+ 41\% vs. control) and VU0092273 ( $+62 \%$ vs. control) increased GFAP levels in CA1 $24 \mathrm{~h}$ after OGD, but only the effect of VU0092273 was statistically significant. Higher resolution images of the indicated areas (Fig. 3. B1a-B4a) indicate that, after treatment with VU0092273, astrocytes were distributed in overlapping domains, showed morphological changes such as hypertrophy of the cell body and processes (Fig. 3B4a, open arrow), and displayed increased expression of GFAP, conditions that resemble those defined as adaptive reactive astrocytosis (Burda and Sofroniew, 2014; Sofroniew, 2015; Choudhury and Ding, 2016).

Quantitative analysis of IBA1 immunostaining (Fig. 3, C1-C4 and F) showed that the density of microglia in CA1 was not modified $24 \mathrm{~h}$ after OGD. Treatment with DHPG and VU0092273 significantly increased microglia proliferation by $44.5 \%$ and $65 \%$, respectively, in comparison to controls (Fig. 3F). The magnifications of the framed areas of panels C1-C4 (Fig. 3. C1a-C4a) show that in OGD slices treated with DHPG and VU0092273 microglia cells display a morphology typical of reactive microglia (Milligan and Watkins, 2009; Cerbai et al., 2012; Lana et al., 2014). In particular, some microglia cell bodies were enlarged and had amoeboid shape (Fig. 3. C4a, open arrow), highlighted by the dotted lines in the magnification of cell body (Fig. 3 C4b, open arrow), others were rod-shaped (Fig. 3. C3a, white arrow),highlighted by the dotted lines in the magnification of cell body (Fig. 3 C3b, white arrow) and their cellular branches were shorter, de-ramified and twisted. This effect was present, albeit to a lesser extent, in untreated OGD slices (Fig. 3. C2a).

\subsection{The PI3K-Akt signalling pathway mediates the protective effects induced by $m$ Glu 5 receptors}

Allosteric agents have the potential to exert differential effects on downstream signalling, a phenomenon termed functional selectivity (Nickols and Conn, 2014). Interestingly, we have shown previously in rat organotypic hippocampal slices that activation of the phosphatidylinositol 3-kinase (PI3K) signalling pathway with the subsequent activation by phosphorylation of the pro-survival protein kinase Akt are implicated in the neuroprotective mechanisms of DHPG preconditioning (Scartabelli et al., 2008). We tested if the neuroprotective effects evoked by mGlu5 receptor activation with a selective PAM were mediated by this kinase signalling cascade. Fig. 4A shows that the PI3K inhibitor LY294002 at $10 \mu \mathrm{M}$ prevented the neuroprotective effects of both $1 \mu \mathrm{M}$ DHPG and $10 \mathrm{nM}$ VU0092273, indicating that PI3K activation is involved in the mechanisms following mGlu5 activation. To examine the downstream effectors of PI3K activation, we used phosphospecific antibodies to measure the relative levels of the phosphorylated, active form of Akt and of the phosphorylated, inactive form of GSK3- $\beta$ in hippocampal slices $24 \mathrm{~h}$ after OGD (Fig. 4B and C). Exposure to OGD induced a marked reduction in the phosphorylation of both Akt (by $68 \%$ ) and GSK3- $\beta$ (by $60 \%$ ), that was completely prevented by co- 

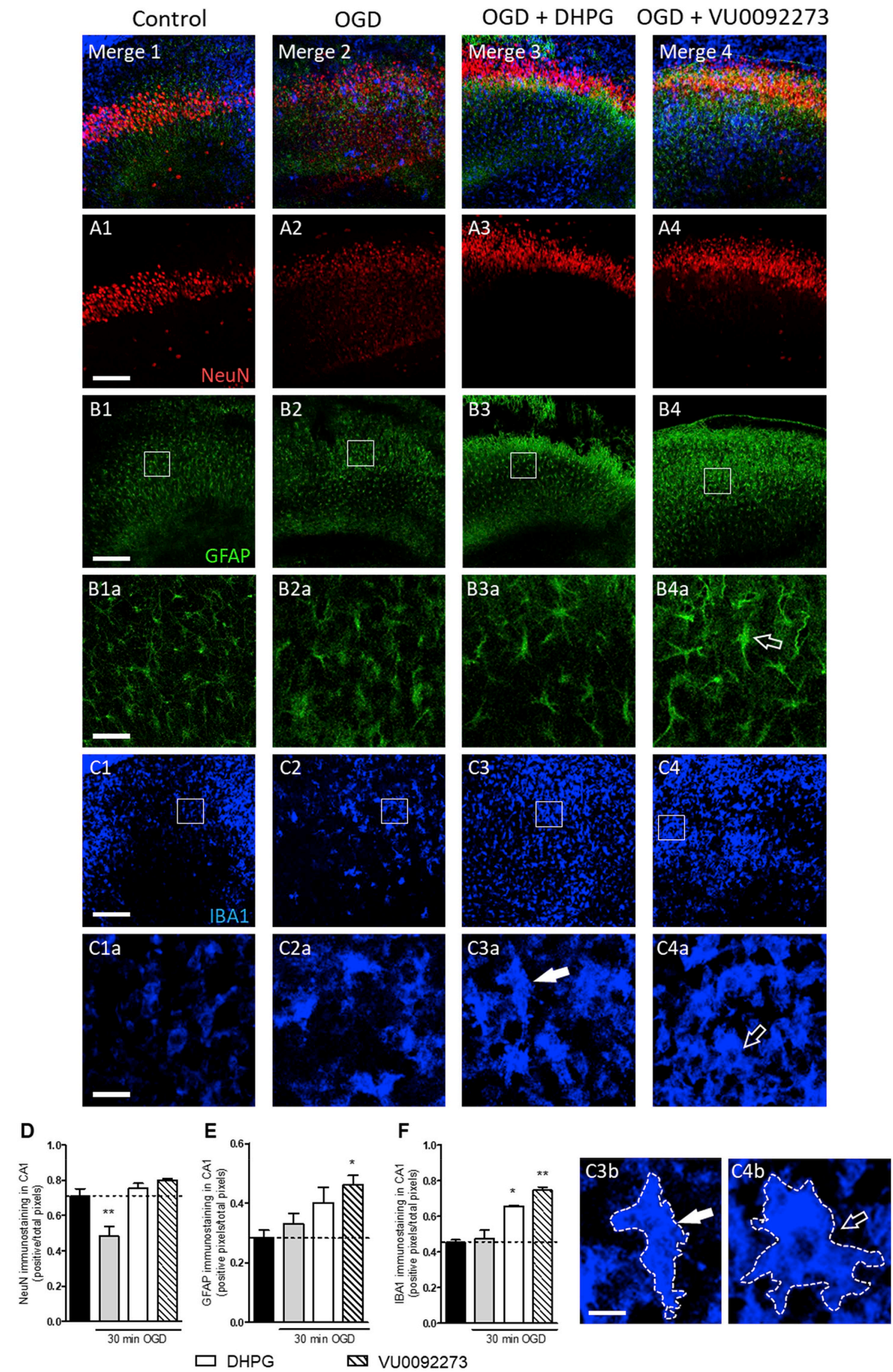


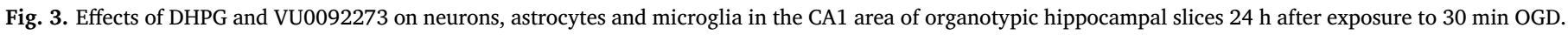

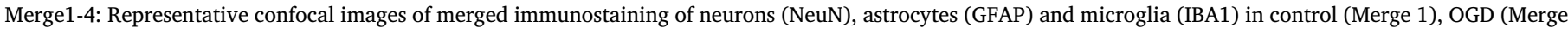

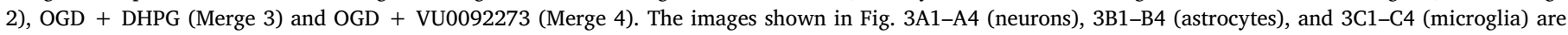

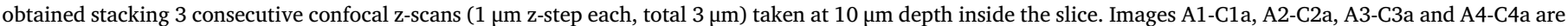

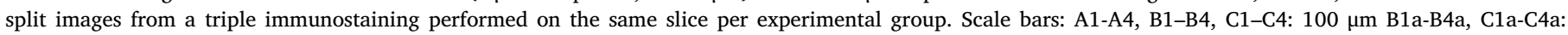

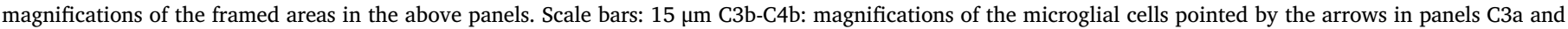

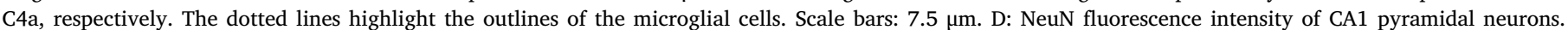

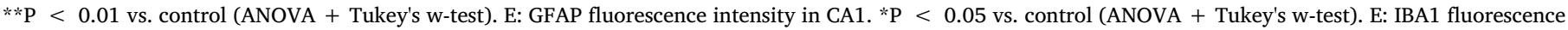

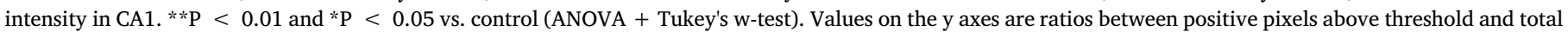
pixels of regions of interest and represent the mean \pm SEM of at least three experiments performed in quadruplicate.

incubation with the mGlu5 PAM VU0092273 (10 nM) but not the mGlu1 PAM VU0483605 $(1 \mu \mathrm{M})$. The PI3K inhibitor LY294002 at $10 \mu \mathrm{M}$ was able to revert the effects of PAM VU0092273, indicating that the mGlu5-mediated increases in the phosphorylation of Akt and GSK3$\beta$ requires activation of the PI3K-Akt cascade.

\subsection{Activation of mGlu5 receptors prevents the down-regulation of the GluA2 AMPA receptor subunit induced by OGD}

We have previously shown that OGD induces a down-regulation in the protein expression of the GluA2 subunit of AMPA receptors in organotypic hippocampal slices prior to CA1 pyramidal cell death (Llorente et al., 2015). This phenomenon has been designated as the AMPA receptor 'molecular switch', that is triggered by cerebral ischemia and other neurological disorders and leads to the formation of GluA2-lacking $\mathrm{Ca}^{2+}$-permeable AMPA receptor channels and neurodegeneration in cells destined to die (Pellegrini-Giampietro et al., 1997; Liu et al., 2006; Henley and Wilkinson, 2016; Koszegi et al., 2017). Twelve hours after OGD, a time point preceding CA1 neuronal death, Western blot analysis confirmed that the protein expression of the

A

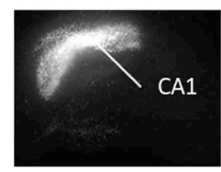

$30 \mathrm{~min}$ OGD

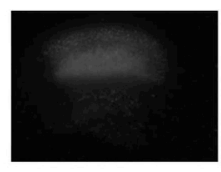

$30 \mathrm{~min} O G D+$ $10 \mathrm{nM}$ VU00922735

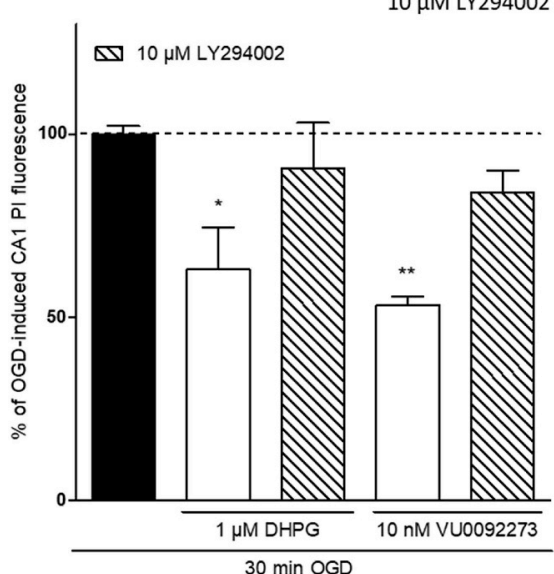

B
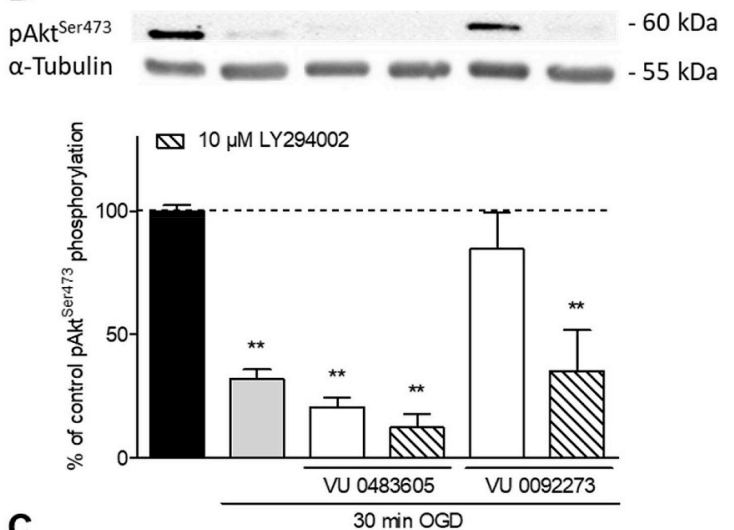

C
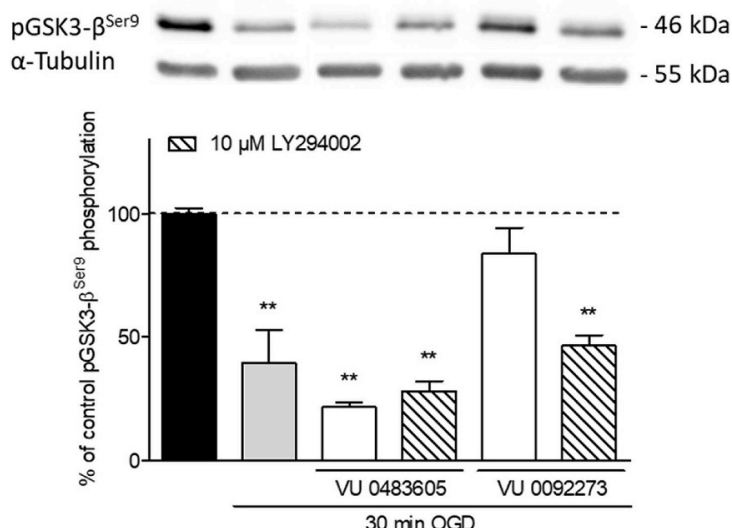

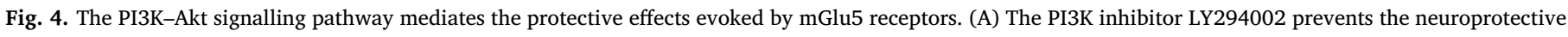

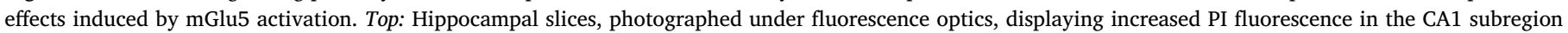

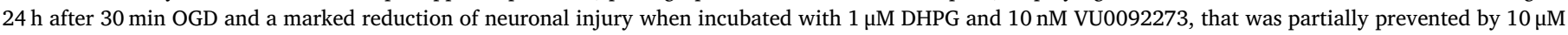

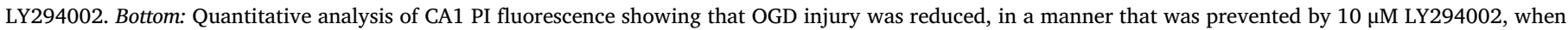

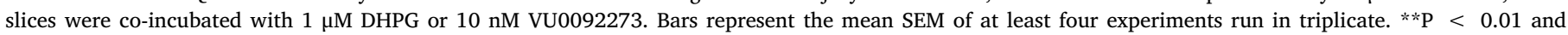

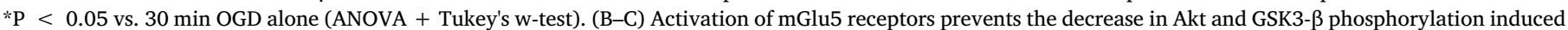

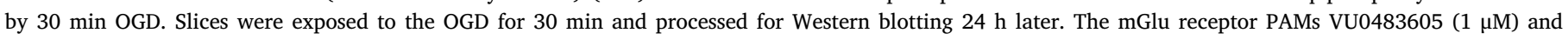

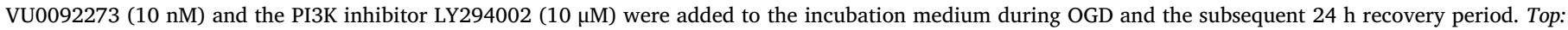

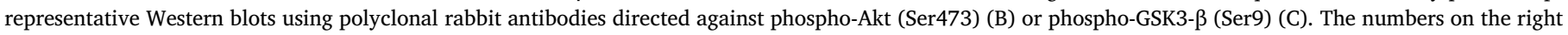

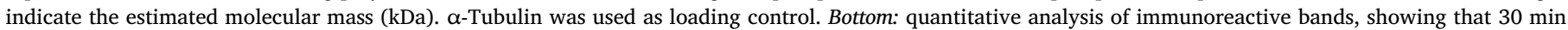

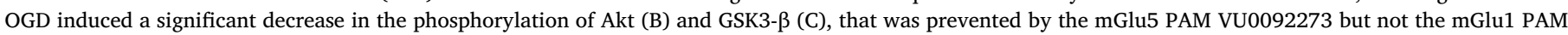

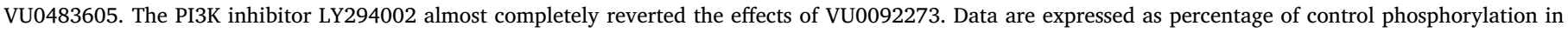
control untreated slices. Bars represent the mean \pm SEM of three experiments. ${ }^{* *} \mathrm{P}<0.01$ vs. control (ANOVA + Tukey's $\mathrm{w}$ test). 
A

$\mathrm{Oh}$

Drugs

$12 \mathrm{~h}$

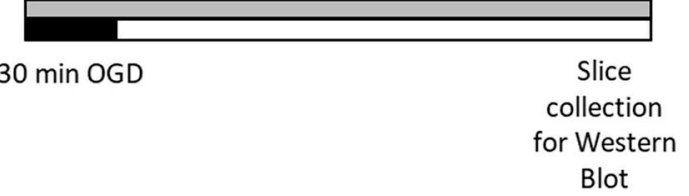

B
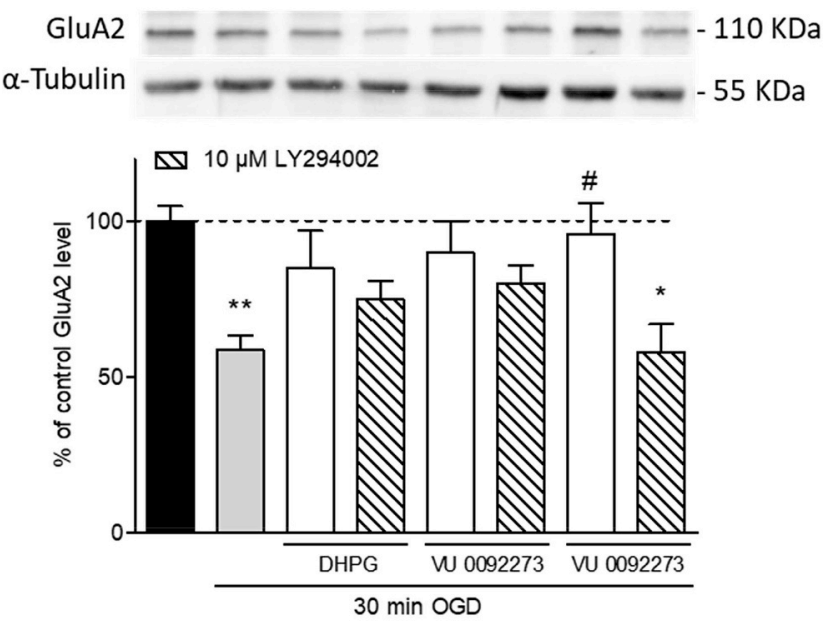

C

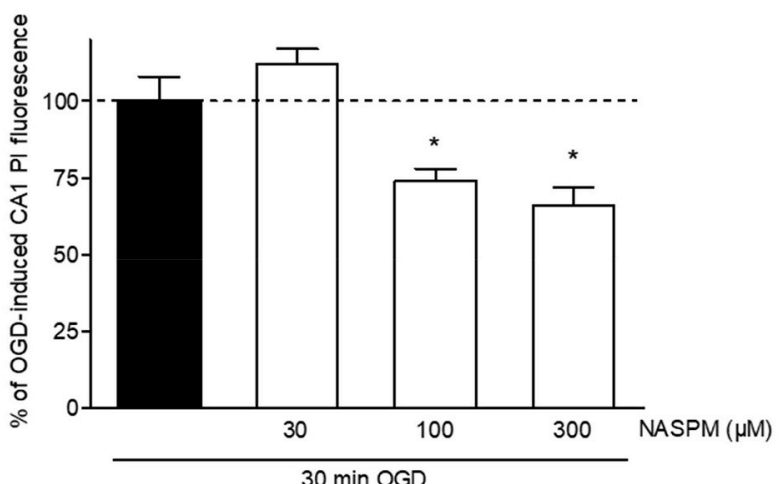

Fig. 5. Effects of mGlu5 receptor activation on the expression of GluA2-lacking AMPA receptors in organotypic hippocampal slices exposed to OGD. The experiments were carried out and drugs were added as shown in Fig. 5A. (A) Schematic diagram showing that slices were incubated with drug during $30 \mathrm{~min}$ OGD and the subsequent recovery period of $12 \mathrm{~h}$. (B) Activation of mGlu5 receptors prevents the down-regulation of the GluA2 AMPA receptor subunit following OGD via the PI3K-Akt signalling pathway. Slices were exposed to OGD for $30 \mathrm{~min}$ and processed for Western blotting $12 \mathrm{~h}$ later. Top: A representative Western blot using a polyclonal goat antibody directed against GluA2. $\alpha$-Tubulin was used as loading control. The numbers on the right indicate the estimated molecular mass $(\mathrm{kDa})$. Bottom: quantitative analysis of immunoreactive bands, showing that 30 min OGD induced $12 \mathrm{~h}$ later a significant decrease in the expression of GluA2, that was significantly prevented by the mGlu5 PAM VU0092273. The PI3K inhibitor LY294002 reverted the effects of VU0092273. Data are expressed as percentage of GluA2 levels in control untreated slices. Bars represent the mean \pm SEM of three experiments. ${ }^{*} \mathrm{P} P 0.01$ vs. control (ANOVA + Tukey's w test). \#P $<0.05$ vs. OGD alone. (C) NASPM attenuates OGD injury in a dose-dependent manner in organotypic hippocampal slices. Data are expressed as percentage of OGD-induced PI fluorescence in CA1 as observed $24 \mathrm{~h}$ after OGD. Values represent the mean \pm SEM of at least three experiments performed in quadruplicate. $* \mathrm{P}<0.05$ vs. 30 min OGD alone (ANOVA + Tukey's w-test). crucial GluA2 subunit that confers $\mathrm{Ca}^{2+}$ impermeability to AMPA receptor channels, was down regulated by $41 \%$ (Fig. 5A). The formation of GluA2-lacking AMPA receptors leading to neurodegeneration in our hippocampal slices was confirmed using NASPM, a selective blocker of $\mathrm{Ca}^{2+}$-permeable AMPA channels, that was able to dose-dependently attenuate the neuronal injury observed $24 \mathrm{~h}$ after OGD (Fig. 5B).

Because activation of group I mGlu receptors has been shown to reverse the formation of $\mathrm{Ca}^{2+}$-permeable AMPA channels in cocainetreated mice ex vivo (Bellone and Lüscher, 2006), we explored whether activation of mGlu receptors in our hippocampal slices could modify the changes in expression of AMPA receptor subunits observed following OGD. Interestingly however, the down-regulation of the GluA2 protein induced by OGD could be prevented only by the mGlu5 PAM VU0092273, suggesting that mGlu5 activation is responsible for the deleterious AMPA molecular switch that leads to OGD injury. We also examined whether the PI3K/Akt pathway could mediate the effects of mGlu receptor activation on the down-regulation of the GluA2 subunit induced by OGD. Interestingly, the PI3K inhibitor LY294002 $(10 \mu \mathrm{M})$ was able to prevent the reversion of the GluA2 down-regulation induced by the mGlu5 PAM VU0092273, but not that induced by DHPG and the mGlu1 PAM VU0483605 (Fig. 5A).

\section{Discussion}

In this study, we show that mGlu5 receptor exerts neuroprotective effects against OGD-induced CA1 injury in rat organotypic hippocampal slices. Using the non-selective group I mGlu agonist DHPG and the mGlu5 selective positive allosteric modulator (PAM) VU0092273 we observed significant reductions in OGD-induced CA1 injury. In contrast the mGlu1 selective PAM VU0483605 had no protective effect. Moreover, the protective actions of DHPG and VU0092273 were blocked by the mGlu5 antagonist MPEP, but not by the mGlu1 antagonist LY367385.

Immunohistochemistry revealed that DHPG and VU0092273 increased the viability of CA1 neurons exposed to OGD and enhanced glia responses, as demonstrated by the hypertrophy of astrocytes processes, and by the amoeboid-shape of microglia cell bodies. It is known that the response of astrocytes to brain injury are different in space and time and can have immediate and positive effects on cell survival or longterm delayed influence, affecting recovery. Indeed, it is still a matter of debate whether reactive astrocytes are harmful or beneficial, since both types of effects have been observed (Alilain et al., 2011; Voskuhl et al., 2009). Different subtypes of reactive astrocytes, such as A1 and A2, differ in their functions, depending on the nature, localization, and time after injury (Liddelow and Barres, 2017). Our results suggest that mGluR5 expressed in astrocytes (Arizono et al., 2014) could stimulate their positive effects contributing to neuroprotection. Moreover, this astrocyte activation also correlates with microglial activation, a mechanism that can contribute to identification and elimination of cellular debris and neutrophils (Neumann et al., 2008; Milligan and Watkins, 2009; Cerbai et al., 2012; Lana et al., 2014) exerting a neuroprotective effect. Indeed, although microglia has long been considered a major contributor of postischemic inflammation, our data are in accordance with the growing evidence that the inflammatory response may be important for tissue repair (Madinier et al., 2009). It has been recently demonstrated that while M1-type microglia release proinflammatory and cytotoxic mediators, M2-type microglia can dampen inflammation and promote tissue regeneration (Chawla et al., 2011). These data are consistent with the concept that reactive gliosis may contribute to decrease of neuronal vulnerability (Burda and Sofroniew, 2014; Pekny and Pekna, 2014; Lana et al., 2016). mGluRs are expressed on astrocytes and microglia and their activation under pathophysiological conditions may shift microglia towards a pro-repair phenotype (Loane et al., 2012; Loane et al., 2014). Further investigations are needed to understand the mechanism underlying the mGluR5 involvement in astrocytes/microglia activation. 
Positive allosteric modulators of mGluR5 show functional selectivity i.e. they have the potential to selectively activate distinct downstream signalling pathway (Nickols and Conn, 2014). Here we investigated the possible role of the PI3K signalling, one of the pathways activated by group I mGluRs (Rong et al., 2003). Moreover, PI3K signalling leads to Akt activation, which is well established to have neuroprotective actions (Datta et al., 1999; Kandel and Hay, 1999).

Consistent with this, the PI3K inhibitor LY294002 completely abolished the neuroprotective effects of DHPG and VU0092273, suggesting the crucial role of the mGluR5-PI3K-Akt signalling pathway in their mechanism of action. Analysis of active phosphorylated Akt (pAkt) showed that pAkt was activated by VU0092273 but not the mGlu1 PAM VU0483605, indicating that activation of PI3K signalling pathway is mGlu5 selective. Moreover, the pro-apoptotic kinase GSK3 $\beta$ (Chen et al., 2004; Linseman et al., 2004) a downstream target of pAkt (Pap and Cooper, 1998; Datta et al., 1999; Yamaguchi et al., 2004), also showed increased phosphorylation. This is important because phosphorylated GSK3 $\beta$ (pGSK3 $\beta$ ) is inactivated (Pap and Cooper, 1998; Datta et al., 1999) so its inhibition can enhance cell survival.

Severe neurological insults trigger a 'molecular switch' that prevents GluA2 AMPA receptor subunit gene expression in cells destined to die (Pellegrini-Giampietro et al., 1997). The GluA2 hypothesis predicts that $\mathrm{Ca}^{2+}$ entry through GluA2-lacking AMPA receptors in neurons that normally express $\mathrm{Ca}^{2+}$-impermeable channels contributes to or causes delayed cell death in response to endogenous glutamate (PellegriniGiampietro et al., 1997; Liu et al., 2006; Henley and Wilkinson, 2016; Koszegi et al., 2017).

We observed the GluA2 down-regulation $12 \mathrm{~h}$ after OGD injury, revealing that this phenomenon precedes cell death, which occurs $24 \mathrm{~h}$ later. Importantly, only mGluR5 PAM significantly prevents the GluA2 down-regulation induced by OGD injury. Moreover, when we measured GluA2 protein levels in presence of PI3K inhibitor, the prevention of GluA2 down-regulation was blocked when mediated by mGluR5 PAM, suggesting that the mGluR5 triggers the GluA2 up-regulation through the activation of PI3K signalling pathway, known to be also involved in activation of nuclear transcription factors, that regulate the expression of a large number of genes, critical for survival (Walton and Dragunow, 2000).

Thus, the mGluR5 exerts its neuroprotective effects against OGD injury by activating the PI3K signalling pathway, through which it triggers all the cascades which lead to block apoptotic processes and promote the cell survival, and enhances the GluA2 up-regulation, preventing the excitotoxic events which cause the delayed cell death.

Taken together our data demonstrate that in our OGD/recovery model mGlu5 (i) activates the PI3K-Akt pathway to promote survival; (ii) reduces GluA2 down-regulation which prevents the excytotoxic cascade triggered by the increased $\mathrm{Ca}^{2+}$ influx due to GluA2-lacking AMPA receptors; and (iii) contributes to reactive astrogliosis which can assist in rescuing post-ischemic neurons from OGD-induced cell death. Thus, we propose that selective activation/potentiation of mGlu5 signalling represents a promising strategy for the development of new interventions to reduce or prevent ischemia-induced neuronal death.

\section{Acknowledgements}

We would like to thank Dr. Jeffrey P. Conn (Vanderbilt University Medical Center, Nashville, TN, USA) for the generous gift of VU0483605 and VU0092273. This work was supported by grants from Ente Cassa di Risparmio di Firenze (ECRF 2015.0964) and the University of Florence. D.L. was a recipient of a post-doctoral fellowship from the University of Florence.

\section{Appendix A. Supplementary data}

Supplementary data to this article can be found online at https:// doi.org/10.1016/j.neuropharm.2019.107810.

\section{References}

Abe, T., Sugihara, H., Nawa, H., Shigemoto, R., Mizuno, N., Nakanishi, S., 1992. Molecular characterization of a novel metabotropic glutamate receptor mGluR5 coupled to inositol phosphate/Ca2 + signal transduction. J. Biol. Chem. 267 (19), 13361-13368.

Alilain, W.J., Horn, K.P., Hu, H., Dick, T.E., Silver, J., 2011. Functional regeneration of respiratory pathways after spinal cord injury. Nature 475 (7355), 196-200. https:// doi.org/10.1038/nature10199.

Arizono, M., Bannai, H., Mikoshiba, K., 2014. Imaging mGluR5 dynamics in astrocytes using quantum dots. Curr. Protoc. Neurosci. 66https://doi.org/10.1002/ 0471142301.ns0221s66. 2.21.1-2.21.18.

Bellone, C., Lüscher, C., 2006. Cocaine triggered AMPA receptor redistribution is reversed in vivo by mGluR-dependent long-term depression. Nat. Neurosci. 9 (5), 636-641. https://doi.org/10.1038/nn1682.

Bignami, A., Eng, L.F., Dahl, D., Uyeda, C.T., 1972. Localization of the glial fibrillary acidic protein in astrocytes by immunofluorescence. Brain Res. 43 (2), 429-435.

Buchan, A.M., Pulsinelli, W.A., 1990. Septo-hippocampal deafferentation protects CA1 neurons against ischemic injury. Brain Res. 512 (1), 7-14. https://doi.org/10.1016/ 0006-8993(90)91163-b.

Burda, J.E., Sofroniew, M.V., 2014. Reactive gliosis and the multicellular response to CNS damage and disease. Neuron 81 (2), 229-248. https://doi.org/10.1016/j.neuron. 2013.12.034.

Cerbai, F., Lana, D., Nosi, D., Petkova-Kirova, P., Zecchi, S., Brothers, H.M., Wenk, G.L., Giovannini, M.G., 2012. The neuron-astrocyte-microglia triad in normal brain ageing and in a model of neuroinflammation in the rat hippocampus. PLoS One 7 (9), e45250. https://doi.org/10.1371/journal.pone.0045250.

Chawla, A., Nguyen, K.D., Goh, Y.P., 2011. Macrophage-mediated inflammation in metabolic disease. Nat. Rev. Immunol. 11 (11), 738-749. https://doi.org/10.1038/ nri3071.

Chen, G., Bower, K.A., Ma, C., Fang, S., Thiele, C.J., Luo, J., 2004. Glycogen synthase kinase 3beta (GSK3beta) mediates 6-hydroxydopamine-induced neuronal death. FASEB J. 18 (10), 1162-1164. https://doi.org/10.1096/fj.04-1551fje.

Cho, H.P., Garcia-Barrantes, P.M., Brogan, J.T., Hopkins, C.R., Niswender, C.M., Rodriguez, A.L., Venable, D.F., Morrison, R.D., Bubser, M., Daniels, J.S., Jones, C.K., Conn, P.J., Lindsley, C.W., 2014. Chemical modulation of mutant mGlu1 receptors derived from deleterious GRM1 mutations found in schizophrenics. ACS Chem. Biol. 9 (10), 2334-2346. https://doi.org/10.1021/cb500560h.

Choudhury, G.R., Ding, S., 2016. Reactive astrocytes and therapeutic potential in focal ischemic stroke. Neurobiol. Dis. 85, 234-244. https://doi.org/10.1016/j.nbd.2015. 05.003 .

Christopoulos, A., Kenakin, T., 2002. G protein-coupled receptor allosterism and complexing. Pharmacol. Rev. 54 (2), 323-374.

Conn, P.J., Pin, J.P., 1997. Pharmacology and functions of metabotropic glutamate receptors. Annu. Rev. Pharmacol. Toxicol. 37, 205-237. https://doi.org/10.1146/ annurev.pharmtox.37.1.205.

Conn, P.J., Christopoulos, A., Lindsley, C.W., 2009. Allosteric modulators of GPCRs: a novel approach for the treatment of CNS disorders. Nat. Rev. Drug Discov. 8 (1), 41-54. https://doi.org/10.1038/nrd2760.

Datta, S.R., Brunet, A., Greenberg, M.E., 1999. Cellular survival: a play in three Akts. Genes Dev. 13 (22), 2905-2927. https://doi.org/10.1101/gad.13.22.2905.

Gerace, E., Landucci, E., Scartabelli, T., Moroni, F., Pellegrini-Giampietro, D.E., 2012. Rat hippocampal slice culture models for the evaluation of neuroprotective agents. Methods Mol. Biol. 846, 343-354. https://doi.org/10.1007/978-1-61779-536-7_29.

Gerace, E., Landucci, E., Totti, A., Bani, D., Guasti, D., Baronti, R., Moroni, F., Mannaioni, G., Pellegrini-Giampietro, D.E., 2016. Ethanol toxicity during brain development: alterations of excitatory synaptic transmission in immature organotypic hippocampal slice cultures. Alcohol Clin. Exp. Res. 40 (4), 706-716. https://doi.org/10.1111/acer. 13006.

Henley, J.M., Wilkinson, K.A., 2016. Synaptic AMPA receptor composition in development, plasticity and disease. Nat. Rev. Neurosci. (6), 337-350. https://doi.org/10. 1038/nrn.2016.37.

Imai, Y., Ibata, I., Ito, D., Ohsawa, K., Kohsaka, S., 1996. A novel gene iba1 in the major histocompatibility complex class III region encoding an EF hand protein expressed in a monocytic lineage. Biochem. Biophys. Res. Commun. (224), 855-862.

Kandel, E.S., Hay, N., 1999. The regulation and activities of the multifunctional serine/ threonine kinase Akt/PKB. Exp. Cell Res. 253 (1), 210-229. https://doi.org/10.1006/ excr.1999.4690.

Koszegi, Z., Fiuza, M., Hanley, J.G., 2017. Endocytosis and lysosomal degradation of GluA2/3 AMPARs in response to oxygen/glucose deprivation in hippocampal but not cortical neurons. Sci. Rep. 7 (1), 12318. https://doi.org/10.1038/s41598-01712534-w.

Lana, D., Iovino, L., Nosi, D., Wenk, G.L., Giovannini, M.G., 2016. The neuron-astrocytemicroglia triad involvement in neuroinflammation mechanisms in the CA3 hippocampus of memory-impaired aged rats. Exp. Gerontol. 83, 71-88. https://doi.org/10 1016/j.exger.2016.07.011.

Lana, D., Melani, A., Pugliese, A.M., Cipriani, S., Nosi, D., Pedata, F., Giovannini, M.G., 2014. The neuron-astrocyte-microglia triad in a rat model of chronic cerebral hypoperfusion: protective effect of dipyridamole. Front. Aging Neurosci. 6, 322. https://doi.org/10.3389/fnagi.2014.00322.

Landucci, E., Lattanzi, R., Gerace, E., Scartabelli, T., Balboni, G., Negri, L., PellegriniGiampietro, D.E., 2016. Prokineticins are neuroprotective in models of cerebral ischemia and ischemic tolerance in vitro. Neuropharmacology 108, 39-48. https:// doi.org/10.1016/j.neuropharm.2016.04.043.

Law, H.C., Szeto, S.S., Quan, Q., Zhao, Y., Zang, Z., Krakovska, O., Lui, L.T., Zheng, C., 
Lee, S.M., Sin, K.W., Wang, Y., Chu, I.K., 2017. Characterization of the molecular mechanisms underlying the chronic phase of stroke in a cynomolgus monkey model of induced cerebral ischemia. J. Proteome Res. 16 (3), 1150-1166. https://doi.org/ 10.1021/acs.jproteome.6b00651.

Liddelow, S.A., Barres, B.A., 2017. Reactive astrocytes: production, function, and therapeutic potential. Immunity 46 (6), 957-967. https://doi.org/10.1016/j.immuni. 2017.06.006.

Linseman, D.A., Butts, B.D., Precht, T.A., Phelps, R.A., Le, S.S., Laessig, T.A., Bouchard, R.J., Florez-McClure, M.L., Heidenreich, K.A., 2004. Glycogen synthase kinase-3bet phosphorylates Bax and promotes its mitochondrial localization during neuronal apoptosis. J. Neurosci. 24 (44), 9993-10002. https://doi.org/10.1523/JNEUROSCI 2057-04.2004.

Liu, B., Liao, M., Mielke, J.G., Ning, K., Chen, Y., Li, L., El-Hayek, Y.H., Gomez, E., Zukin, R.S., Fehlings, M.G., Wan, Q., 2006. Ischemic insults direct glutamate receptor subunit 2-lacking AMPA receptors to synaptic sites. J. Neurosci. 26 (20), 5309-5319. https://doi.org/10.1523/JNEUROSCI.0567-06.2006.

Loane, D.J., Stoica, B.A., Faden, A.I., 2012. Metabotropic glutamate receptor-mediated signaling in neuroglia Wiley Interdiscip. Rev. Membr. Transp. Signal 1 (2), 136-150. https://doi.org/10.1002/wmts.30.

Loane, D.J., Stoica, B.A., Tchantchou, F., Kumar, A., Barrett, J.P., Akintola, T., Xue, F., Conn, P.J., Faden, A.I., 2014. Novel mGluR5 positive allosteric modulator improves functional recovery, attenuates neurodegeneration, and alters microglial polarization after experimental traumatic brain injury. Neurotherapeutics 11 (4), 857-869. https://doi.org/10.1007/s13311-014-0298-6.

Llorente, I.L., Landucci, E., Pellegrini-Giampietro, D.E., Fernández-López, A., 2015. Glutamate receptor and transporter modifications in rat organotypic hippocampal slice cultures exposed to oxygen-glucose deprivation: the contribution of cyclooxygenase-2. Neuroscience 292, 118-128. https://doi.org/10.1016/j.neuroscience. 2015.02.040.

Madinier, A., Bertrand, N., Mossiat, C., Prigent-Tessier, A., Beley, A., Marie, C., Garnier, P., 2009. Microglial involvement in neuroplastic changes following focal brain ischemia in rats. PLoS One 4 (12), e8101. https://doi.org/10.1371/journal.pone. 0008101.

Meli, E., Picca, R., Attucci, S., Cozzi, A., Peruginelli, F., Moroni, F., Pellegrini-Giampietro, D.E., 2002. Activation of mGlu1 but not mGlu5 metabotropic glutamate receptors contributes to postischemic neuronal injury in vitro and in vivo. Pharmacol. Biochem. Behav. 73 (2), 439-446. https://doi.org/10.1016/s0091-3057(02)00834-1.

Middeldorp, J., Hol, E.M., 2011. GFAP in health and disease. Prog. Neurobiol. 93 (3), 421-443. https://doi.org/10.1016/j.pneurobio.2011.01.005.

Milligan, E.D., Watkins, L.R., 2009. Pathological and protective roles of glia in chronic pain. Nat. Rev. Neurosci. 10 (1), 23-36. https://doi.org/10.1038/nrn2533.

Neumann, J., Sauerzweig, S., Ronicke, R., Gunzer, F., Dinkel, K., Ullrich, O., Gunzer, M., Reymann, K.G., 2008. Microglia cells protect neurons by direct engulfment of invading neutrophil granulocytes: a new mechanism of CNS immune privilege. J. Neurosci. 28 (23), 5965-5975. https://doi.org/10.1523/JNEUROSCI.0060-08.2008.

Nickols, H.H., Conn, P.J., 2014. Development of allosteric modulators of GPCRs for treatment of CNS disorders. Neurobiol. Dis. 61, 55-71. https://doi.org/10.1016/j. nbd.2013.09.013.

Noetzel, M.J., Jones, C.K., Conn, P.J., 2012. Emerging approaches for treatment of schizophrenia: modulation of glutamatergic signaling. Discov. Med. 14 (78), 335-343.

Pap, M., Cooper, G.M., 1998. Role of glycogen synthase kinase-3 in the phosphatidylinositol 3-Kinase/Akt cell survival pathway. J. Biol. Chem. 273 (32), 19929-19932. https://doi.org/10.1074/jbc.273.32.19929.

Pekny, M., Pekna, M., 2014. Astrocyte reactivity and reactive astrogliosis: costs and benefits. Physiol. Rev. 94 (4), 1077-1098. https://doi.org/10.1152/physrev.00041. 2013.

Pellegrini-Giampietro, D.E., 2003. The distinct role of mGlu1 receptors in post-ischemic neuronal death. Trends Pharmacol. Sci. 24 (9), 461-470. https://doi.org/10.1016/ S0165-6147(03)00231-1.

Pellegrini-Giampietro, D.E., Gorter, J.A., Bennett, M.V., Zukin, R.S., 1997. The GluR2 (GluR-B) hypothesis: $\mathrm{Ca}(2+)$-permeable AMPA receptors in neurological disorders. Trends Neurosci. 20 (10), 464-470.

Pellegrini-Giampietro, D.E., Peruginelli, F., Meli, E., Cozzi, A., Albani-Torregrossa, S., Pellicciari, R., Moroni, F., 1999. Protection with metabotropic glutamate 1 receptor antagonists in models of ischemic neuronal death: time-course and mechanisms. Neuropharmacology 38 (10), 1607-1619.

Rong, R., Ahn, J.Y., Huang, H., Nagata, E., Kalman, D., Kapp, J.A., Tu, J., Worley, P.F., Snyder, S.H., Ye, K., 2003. PI3 kinase enhancer-Homer complex couples mGluRI to PI3 kinase, preventing neuronal apoptosis. Nat. Neurosci. 6 (11), 1153-1161. https://doi.org/10.1038/nn1134.

Scartabelli, T., Gerace, E., Landucci, E., Moroni, F., Pellegrini-Giampietro, D.E., 2008. Neuroprotection by group I mGlu receptors in a rat hippocampal slice model of cerebral ischemia is associated with the PI3K-Akt signaling pathway: a novel postconditioning strategy? Neuropharmacology 55 (4), 509-516. https://doi.org/10. 1016/j.neuropharm.2008.06.019.

Sofroniew, M.V., 2015. Astrocyte barriers to neurotoxic inflammation. Nat. Rev. Neurosci. 16 (5), 249-263. https://doi.org/10.1038/nrn3898.

Voskuhl, R.R., Peterson, R.S., Song, B., Ao, Y., Morales, L.B., Tiwari-Woodruff, S., Sofroniew, M.V., 2009. Reactive astrocytes form scar-like perivascular barriers to leukocytes during adaptive immune inflammation of the CNS. J. Neurosci. 29 (37), 11511-11522. https://doi.org/10.1523/JNEUROSCI.1514-09.2009.

Walton, M.R., Dragunow, I., 2000. Is CREB a key to neuronal survival? Trends Neurosci. 23 (2), 48-53.

Werner, C.G., Scartabelli, T., Pancani, T., Landucci, E., Moroni, F., Pellegrini-Giampietro, D.E., 2007. Differential role of mGlu1 and mGlu5 receptors in rat hippocampal slice models of ischemic tolerance. Eur. J. Neurosci. 25 (12), 3597-3604. https://doi.org/ 10.1111/j.1460-9568.2007.05614.x.

Yamaguchi, K., Lee, S.H., Eling, T.E., Baek, S.J., 2004. Identification of nonsteroidal antiinflammatory drug-activated gene (NAG-1) as a novel downstream target of phosphatidylinositol 3-kinase/AKT/GSK-3beta pathway. J. Biol. Chem. 279 (48), 49617-49623. https://doi.org/10.1074/jbc.M408796200. 